\title{
Predictors of constrictive pericarditis after tuberculous pericarditis
}

\author{
Piya K Suwan, Sumontip Potjalongsilp
}

\begin{abstract}
Objective-To identify features which predict the subsequent development of constrictive pericarditis from acute or subacute tuberculous (TB) pericarditis. Setting-Tertiary referral centre, chest hospital.

Patients-The records of 16 consecutive patients in whom acute or subacute TB pericarditis was diagnosed between 1988 and 1990 at a chest hospital were reviewed. These records included a follow up of at least 12 months.

Results-During a follow up of $14 \cdot 2$ (1230) months, 8 patients had constrictive pericarditis diagnosed by cardiac catheterisation or by inspection at the time of operation (group A). There was no evidence of constriction in the other eight patients (group B). There was no significant difference between the two groups in the type or duration of symptoms of TB pericarditis before admission or the volume and characteristics of pericardial fluid obtained at hospital admission. Clinical features of cardiac tamponade on admission correlated closely with the subsequent development of constrictive pericarditis requiring pericardectomy $(7 / 8 v 2 / 8 ; P=0.01)$, despite the fact that the signs of tamponade resolved completely after pericardiocentesis.

Conclusion-The findings suggest that cardiac tamponade in the early clinical stage of TB pericarditis is the most predictive factor of subsequent constrictive pericarditis. The degree of fibrosis of pericardium when treatment starts may be the most important determinant of whether or not constriction develops.
\end{abstract}

(Br Heart f 1995;73:187-189)

Keywords: constrictive pericarditis, tuberculous pericarditis

Cardiology

Department, Central

Chest Hospital, Nonthaburi, Thailand

P Kasem-Suwan P Kasem-Suwan

Correspondence to:

Correspondence to:

Minneapolis Heart Institute,

920 East 28 th Street,

920 East 28 th Street, MN 55407, USA.

Accepted for publication 31 August 1994
Constrictive pericarditis is one of the most serious sequelae of tuberculous (TB) pericarditis. It occurs in $30-50 \%$ of patients despite prompt antituberculous treatment. ${ }^{1-5}$ Morbidity and mortality are significantly higher in those patients in whom pericardial inflammation progresses to a chronic noncompliant fibrotic lesion. Constriction not only produces symptoms of systemic venous congestion but may also cause deterioration of myocardial performance. The increased safety of pericardectomy means that it is now indicated early after the onset of the condition to prevent the deterioration of cardiac function. ${ }^{67}$ The early prediction of those likely to progress to constriction might enable prompt intervention to alter the course of the condition.

We collected data on the clinical features and laboratory findings of patients admitted with active (acute or subacute) TB pericarditis, in whom there was enough pericardial fluid for analysis. We looked for features that may predict the subsequent development of constrictive pericarditis.

\section{Patients}

We reviewed the records of 16 consecutive patients who had a discharge diagnosis of acute or subacute TB pericarditis between 1988 and 1990 at the Central Chest Hospital, Thailand. The criteria judged to be adequate for the diagnosis in these cases were signs of caseous necrosis in specimens of pericardium taken at operation or clinical evidence of pericarditis with a pericardial effusion on the echocardiogram, a strong positive $(>10 \mathrm{~mm})$ tuberculin test, no evidence for other causes of pericarditis and effusion (especially for malignant pericardial effusion), and a good clinical response to antituberculosis treatment. All patients had percutaneous needle aspiration of pericardial effusions under echocardiographic guidance. The clinical features, laboratory findings, and pericardial fluid profiles were collected and evaluated. Patients who had diagnosis of TB pericarditis but presented in the late stage of chronic constrictive pericarditis were not enrolled in this study. All patients had antituberculosis treatment with isoniazid, ethambutol, and rifampicin for 12 months from diagnosis. Ten of 16 patients had been treated with prednisolone $(1 \mathrm{mg} / \mathrm{kg} /$ day) for 3 months and eight of 16 patients underwent pericardectomy during the follow up period.

\section{STATISTICAL ANALYSIS}

Data were expressed as mean (SD) or range. We used Student's $t$ test to compare the differences between the two groups in continuous variables and Fisher's exact test for categorical values. A $P$ value of $<0.05$ was regarded as significant.

\section{Results}

During a mean follow up of $14 \cdot 2(12-30)$ months eight patients (group A) had constrictive pericarditis diagnosed by cardiac 
catheterisation or by inspection at operation. All underwent surgical pericardectomy with satisfactory results. In all eight the pericardium was thick and there was chronic inflammation suggesting tuberculosis. In three cases there was caseous necrosis. None of the other eight patients (group B) showed evidence of constriction during antituberculosis treatment.

Table 1 shows clinical features in both groups. There were six male and two female patients in each group and the mean age on admission was $34.6(7 \cdot 0)$ and $47 \cdot 1(12.9)$ (group A and B respectively). In both groups the most common presenting symptoms were fever and dyspnoea (65\% and $80 \%)$. Other symptoms were chest pain, cough, leg oedema, and anorexia and malaise.

Signs of cardiac tamponade - that is, jugular venous distension, leg oedema, hepatomegaly, or pulsus paradoxus-were significantly more common in group $\mathrm{A}$ than group $\mathrm{B}(7 / 8 v 2 / 8, \mathrm{P}=0.01)$. All these signs resolved after pericardial fluid had been removed by percutaneous needle aspiration and drainage. Pericardial rub was found in $50 \%$ of both groups. Full blood count, urinalysis, and blood chemistry were similar in the two groups.

Effusion volume, gross appearance, specific gravity, protein content, or cell count were similar in both groups (table 2).

\section{Discussion}

Pericarditis caused by tubercle bacilli is an important cause of pericardial disease in regions where tuberculosis (TB) is still preva-

Table 1 Clinical features of patients in whom constrictive pericarditis (CP) developed during follow-up (Group $A$ ) and in whom it did not (Group B)

\begin{tabular}{llll}
\hline & Group $A(C P)$ & Group $B$ & P value \\
\hline Number of cases: & 8 & 8 & NS \\
$\quad$ Male & 6 & 6 & NS \\
Female & 2 & 2 & NS \\
Age (mean (SD)) & $37(12 \cdot 9)$ & & \\
Symptoms: & & 4 & \\
Fever & 6 & 7 & NS \\
Dyspnoea & 2 & 1 & NS \\
Chest pain & 3 & 2 & NS \\
Cough & 4 & $10(1-24)$ & NS \\
Duration (days) & $7(2-24)$ & & \\
Clinical signs: & & 6 & NS \\
$\quad$ Pyrexia (>37.8 & & & $<0 \cdot 05$ \\
Oedema & 6 & 1 & $<0 \cdot 05$ \\
Ascites & 7 & 1 & $<0 \cdot 05$ \\
Hepatomegaly & 7 & 0 & NS \\
Paradoxical pulse & 5 & 4 & \\
Pericardial rub & 2 & 4 & \\
\hline
\end{tabular}

Table 2 Data on pericardial effusion in patients in whom Constrictive Pericarditis (group A) developed and in whom it did not (group B)

\begin{tabular}{lcc}
\hline Data & Group $A$ & Group $B$ \\
\hline Amount aspirated (ml) & $670 \cdot 5(320-1200)$ & $701 \cdot 2(400-980)$ \\
Straw colour & $6 / 8$ & $6 / 8$ \\
Specific gravity (mean (SD)) & $1027(5 \cdot 3)$ & $1030(3 \cdot 8)$ \\
Protein content (g/dl) & $4 \cdot 0(1 \cdot 0)$ & $4 \cdot 4(0 \cdot 9)$ \\
Total Leucocyte count $\left(10^{\circ} / 1\right)$ & $2 \cdot 6(1 \cdot 0-5 \cdot 8)$ & $2 \cdot 6(1 \cdot 4 \cdot 5 \cdot 8)$ \\
Lymphocyte (\%) & $65 \cdot 1(16 \cdot 1)$ & $71 \cdot 8(15 \cdot 2)$ \\
\hline
\end{tabular}

lent. Patients with acute and subacute infection most frequently present with dyspnoea, cough, weight loss, or fever..$^{1-3}$ At presentation $40-60 \%$ of these patients have dependent oedema, indicating chronic cardiac tamponade. About $30-50 \%$ of patients with chronic constrictive pericarditis need surgical removal of the diseased pericardium. ${ }^{459}$ In some patients prompt treatment with antituberculosis drugs and corticosteroids does not stop constriction developing. ${ }^{10-12}$

TB pericarditis develops in four stages: (1) dry or fibrinous, (2) effusive, (3) absorptive or early fibrous, and (4) constrictive or late fibrous. ${ }^{13}$ Prompt treatment given in the first two stages, may prevent constriction in more than half of patients. ${ }^{1-5}$ Beyond stage 3 the clinical signs and symptoms of cardiac tamponade or congestion appear and surgical intervention is needed. ${ }^{14}$

In cardiac tamponade fluid under increased pressure in the pericardial sac impedes venous return and ventricular diastolic filling. ${ }^{15}$ Clinical examination shows raised systemic venous pressure, tachycardia, dyspnoea, and paradoxical arterial pulse. Various amounts of pericardial fluid can cause clinical tamponade. After penetrating heart injury a few hundred mililitres can cause tamponade, whereas in chronic pericardial effusion cardiac tamponade may not develop until more than several hundred mililitres accumulate. ${ }^{16}$ This is because the pericardium can stretch to allow for the increase in the volume of the pericardial sac without increasing intrapericardial pressure. ${ }^{17}$ A large amount of fluid can slowly accummulate in the normally compliant pericardium with only slightly increased intrapericardial pressure without any clinical evidence of cardiac tamponade. ${ }^{15} 17$ A fibrotic diseased pericardium, however, is less compliant and cardiac tamponade will present earlier. Subsequent pericardial constriction is also more likely to develop.

The results of several large series of patients undergoing pericardectomy for constrictive pericarditis were consistently good. ${ }^{6}$ A low cardiac output syndrome persisted after pericardectomy in a considerable number of patients. This may result from myocardial involvement with the epicardial fibrosis penetrating deeply or from long-standing pericardial restriction causing irreversible atrophic changes in the myocardium. ${ }^{14}$ It is now thought that if pericardial constriction is suspected pericardectomy should be performed early to allow an easier surgical technique with low surgical mortality and preservation of myocardium function. ${ }^{?}$

We found that patients who presented with signs of congestion caused by right-sided or chronic cardiac tamponade were more likely to develop constrictive pericarditis. This group of patients may have early effusive constrictive pericarditis and the clinical signs of increased venous pressure may disappear after pericardial fluid is withdrawn. ${ }^{18}$ At this stage, the pericardium may have lost its compliance and the progressive development of constriction may be irreversible. This process does 
not seem to be arrested effectively by current medical treatment.

In conclusion, in patients with acute or subacute TB pericarditis presenting with pericardial effusion the risk of developing constrictive pericarditis is high if there are any clinical manifestations of cardiac tamponade. These may indicate that the disease was advanced before clinical presentation, and thus before the start of antituberculosis treatment, or possibly that the patient had had previous pericardial disease. Such patients should have early surgical intervention.

We thank Dr P Boreham and Dr J C Pitts-Crick of Cardiology Department, Bristol Royal Infirmary for critical review of this paper; and Professor Tada Chakorn of Central Chest paper; and Professor Tada Chakorn of Cert, Thailand, for his valuable comments.

1 Desai HN. Tuberculous pericarditis: A review of 100 cases. S Afr Med f 1979;55:877-80.

2 Hageman JH, D'Esopo ND, Glenn WL. Tuberculous of the pericardium: A long-term analysis of forty-four proved cases. $N$ Engl $千$ Med 1964;270:327-32.

3 Stang JIG, Kakaza HHS, Gibson DG, et al. Controlled clinical tial of complete open surgical drainage an clinical trial of complete open surgical drainage and of prednisolone in treatment of tuberculous pericarditis
4 Long R, Younes M, Patton N, Hershfield E. Tuberculous pericarditis: Long term outcome in patients who received

5 Sagrista-Sauleda J, Permanger-Miralda G, Soler-Soler J. Tuberculous pericarditis: Ten year experience with a prospective protocol for diagnosis and treatment. $\mathcal{F} \mathrm{Am}$ Coll Cardiol 1988;11:124-8.

6 Wychalis AR, Connolly DC, McGoon DC. Surgical treatment of pericarditis. $\mathcal{F}$ Thorac Cardiovas Surg 1971;62: 608-17.

7 McCaughen BC, Hatzell V, Schaff HV, et al. Early and late results of pericardectomy for constrictive pericarditis. $\mathcal{F}$ Thorac Cardiovas Surg 1985;89:340-50.

8 Fowler NO. Tuberculous pericarditis. $¥ A M A$ 1991;266: 99-103.

9 Gooi HL, Smith JM. Tuberculous pericarditis in Birmingham. Thorax 1978;33:94-6.

10 Ortbals DW, Avioli LV. Tuberculous pericarditis. Arch Intern Med 1979;139:231-4.

11 Rooney J, Crocco JA, Lyons HA. Tuberculous pericarditis. Ann Intern Med 1970;72:73-8.

12 Whyte $M$. Tuberculous pericarditis with rapid progression to constriction: Prompt diagnosis and treatment are needed. $B M F$ 1993;307:1052-4.

13 Fitzgerald Peel AA. Tuberculous pericarditis. Br Heart $\mathcal{f}$ 1948;10:195-222.

14 Carrien AJ, Tyers GFO, Williams EH, Derrick JR. Recent experience with tuberculous pericarditis. Ann Thorac Surg 1980;29:464-8.

15 Fowler NO. Cardiac tamponade: A clinical or echocardiographic diagnosis? Circulation 1993;87:1738-41.

16 Shabetai R, Fowler NO, Guntheroth WG. The hemodynamics of cardiac tamponade and constrictive pericarditis. Am $\mathcal{F}$ Cardiol 1970;26:480-9.

17 Spodick DH. The normal and diseased pericardium: Current concepts of pericardial physiology, diagnosis Current concepts of pericardial physiology, diagnosis
and treatment. $₹ \mathrm{Am}$ Coll Cardiol 1983;1:240-51.

$18 \mathrm{Hancock}$ EW. On the elastic and rigid forms of constrictive pericarditis. Am Heart $\mathcal{1}$ 1980;100:918-24. 